\title{
Efeitos da Terapia Combinada Atorvastatina e Clopidogrel na Biodisponibilidade da Estatina e na Função Plaquetária em Pacientes com Doença Coronária Estável
}

\author{
Luiz Fernando Muniz Pinheiro', Maria Cristina de Oliveira Izar1, Soraia Kani Kasmas', \\ Carolina Nunes França', Simone Cristina Matheus Fischer', Simone Pinto de Melo Barbosa', \\ Gilberto de Nucci², Jaime Ilha², Lu Chi Chen², Antonio Carlos Carvalho', \\ Rui Manoel dos Santos Póvoa ${ }^{1}$, Henrique Tria Bianco ${ }^{1}$, Francisco Antonio Helfenstein Fonseca ${ }^{1}$
}

\section{RESUMO}

Introdução: Atorvastatina $80 \mathrm{mg}$ é recomendada a pacientes portadores de doença coronária para redução de eventos cardiovasculares, havendo controvérsia sobre as interações farmacocinéticas entre doses elevadas das estatinas e uso concomitante de clopidogrel, por compartilharem a mesma via de biotransformação. Este estudo avaliou os efeitos da terapia combinada atorvastatina/clopidogrel na farmacocinética da estatina e função plaquetária em pacientes com doença coronária estável, sob uso crônico e efetivo de estatina. Método: Os pacientes foram admitidos quatro vezes para internação (V1 a V4) em leito-dia. Sete dias (D) antes da primeira internação a estatina em uso foi suspensa. A seguir, receberam atorvastatina $80 \mathrm{mg}$ (D1 a D22) e clopidogrel $75 \mathrm{mg} /$ dia (D8 a D29). Em todas as $\mathrm{V}$ foram obtidas amostras de sangue em jejum para dosagens lipídicas, avaliação da função plaquetária (técnica da placa e cone) e quantificação dos níveis plasmáticos de atorvastatina (cromatografia líquida e espectrometria de massa). Resultados: A suspensão por uma semana da estatina modificou o perfil lipídico $(P<0,05$ vs. basal), ocorrendo rápida melhora de todas as frações lipídicas após atorvastatina $80 \mathrm{mg}(\mathrm{P}<0,005 ; \mathrm{V} 1>\mathrm{V} 2, \mathrm{~V} 3$ e V4). $\mathrm{A}$ adesão plaquetária foi menor com clopidogrel isolado $(P=0,003 ; V 4<V 1, V 2$ e V3), enquanto para a agregação houve menor valor com tratamento combinado atorvastatina/clopidogrel ou clopidogrel isolado comparado aos demais períodos ( $\mathrm{P}<0,0001$; V3 e V4 $<$ V1 e V2). O clopidogrel não modificou as concentrações de atorvastatina. Conclusão: Atorvastatina em alta dose não afetou as

\section{ABSTRACT}

Effects of the Atorvastatin and Clopidogrel

Combination Therapy on Statin Bioavailability and Platelet Function of Patients with Stable Coronary Disease

Background: Atorvastatin $80 \mathrm{mg}$ is recommended in patients with coronary artery disease to reduce cardiovascular events, however, there is controversy regarding the pharmacokinetic interactions between high doses of statins and the concomitant use of clopidogrel, since they share the same biotransformation pathway. This study evaluated the effects of the atorvastatin/clopidogrel combination therapy on the pharmacokinetics of statins and on platelet function of patients with stable coronary artery disease receiving chronic statins. Method: Patients were admitted four times (V1 to V4) to a day-clinic. Statin was discontinued seven days (D) before the first admission. Patients then received atorvastatin $80 \mathrm{mg}$ (D1 to D22) and clopidogrel $75 \mathrm{mg} /$ day (D8 to D29). Fasting blood samples were obtained at all time points for lipid measurements, platelet function tests (cone and plate technique), and quantification of atorvastatin plasma levels (liquid chromatography and mass spectrometry). Results: The discontinuation of statins for one week changed the lipid profile $(P<0.05$ vs. baseline), with an early improvement of all lipid parameters after the administration of atorvastatin $80 \mathrm{mg}(\mathrm{P}<0.005 ; \mathrm{V} 1>\mathrm{V} 2, \mathrm{~V} 3$ and $\checkmark 4)$. Platelet adhesion was lower with clopidogrel alone $(\mathrm{P}=0.003 ; \mathrm{V} 4<\mathrm{V} 1, \mathrm{~V} 2$ and $\mathrm{V} 3)$, whereas platelet aggregation values were lower following the atorvastatin/clopidogrel

1 Disciplina de Cardiologia - Universidade Federal de São Paulo São Paulo, SP, Brasil.

2 Instituto Galeno - Campinas, SP, Brasil.

Correspondência: Luiz Fernando Muniz Pinheiro. Rua Pedro de Toledo, 276 - Vila Clementino - São Paulo, SP, Brasil - CEP 04039-030

E-mail: luizfmpinheiro@uol.com.br

Recebido em: 18/4/2010 • Aceito em: 4/6/2010 
Pinheiro LFM, et al. Efeitos da Terapia Combinada Atorvastatina e Clopidogrel na Biodisponibilidade da Estatina e na Função Plaquetária em Pacientes com Doença Coronária Estável. Rev Bras Cardiol Invasiva. 2010;18(2):185-92.

respostas plaquetárias ao clopidogrel; entretanto, curto período de suspensão da estatina piorou o perfil lipídico e a função plaquetária.

DESCRITORES: Agregação plaquetária. Farmacocinética. Doenças cardiovasculares.

A combinação de clopidogrel com ácido acetilsalicílico tem sido universalmente recomendada a pacientes submetidos a intervenção coronária percutânea com implante de stents. Entretanto, alguns pacientes apresentam resistência às ações antiplaquetárias desses medicamentos. ${ }^{1} \mathrm{~A}$ atividade farmacológica do clopidogrel é mediada pela formação de um metabólito obtido pela ação do citocromo CYP450 isoenzima 3A4, mesmo sítio de biotransformação da atorvastatina, sinvastatina e lovastatina ${ }^{2-5}$; além disso, demonstrações in vitro indicam que o clopidogrel pode influenciar as concentrações da fluvastatina por também inibir a atividade CYP 2 C9. ${ }^{6} \mathrm{O}$ metabólito ativo do clopidogrel, um tiol instável, inibe a agregação plaquetária, formando pontes dissulfídicas com um resíduo cisteína do receptor para adenosina difosfato nas plaquetas. ${ }^{5} \mathrm{O}$ clopidogrel, no entanto, não modifica outros parâmetros da coagulação, em pacientes com síndromes coronárias agudas ${ }^{7}$ ou após revascularização periférica ${ }^{8}$.

A literatura é restrita quanto a relatos das interações entre fármacos. Estudos que compararam estatinas empregaram doses relativamente baixas, que dificilmente atingiram a meta do colesterol de lipoproteína de baixa densidade (LDL-colesterol) $<70 \mathrm{mg} / \mathrm{dl}$, hoje sugeridas para pacientes em prevenção secundária de doença coronária. No entanto, o uso de estatina associada ao clopidogrel no infarto do miocárdio sem supradesnivelamento do segmento ST ou na angina instável influenciou favoravelmente a sobrevida livre de eventos no Global Registry of Acute Coronary Events (registro GRACE). ${ }^{9}$

Especula-se que a magnitude da redução lipídica pela estatina possa ser atenuada pela competição pelo substrato microssomal hepático ou que a resistência ao clopidogrel possa estar associada à menor expressão de seus metabólitos, dependentes, da biotransformação hepática ${ }^{10}$, além do possível efeito de variantes genéticas das enzimas CYP3A4. ${ }^{11}$ Assim, interações farmacocinéticas podem afetar o efeito antiagregante do clopidogrel ou reduzir os efeitos hipolipemiantes e pleiotrópicos da estatina. ${ }^{10,12} \mathrm{~A}$ atorvastatina sofre biotransformação pelo CYP3A4, mas é ativa antes mesmo da metabolização hepática ${ }^{10-13}$, e pode ainda inibir o metabolismo de alguns substratos do CYP3A4, entre os quais o clopidogrel. Estudos recentes demonstraram que a ativação do clopidogrel é complexa, envolven- combination therapy or clopidogrel alone when compared to the other time points $(\mathrm{P}<0.0001 ; \mathrm{V} 3$ and $\mathrm{V} 4<\mathrm{V} 1$ and $\mathrm{V} 2)$. The use of clopidogrel did not affect atorvastatin serum levels. Conclusion: High-dose atorvastatin did not affect platelet responses to clopidogrel, however the short-term statin discontinuation worsened the lipid profile and platelet function.

KEY-WORDS: Platelet aggregation. Pharmacokinetics. Cardiovascular diseases.

do duas etapas sequenciais mediadas por CYP3A, CYP1A2, CYP2C9, CYP2C19 e/ou CYP2B6. ${ }^{13}$

O objetivo primário deste estudo foi avaliar os efeitos da terapia combinada com atorvastatina e clopidogrel na farmacocinética da estatina e na função plaquetária em pacientes com doença coronária estável. Como objetivo secundário, analisamos as modificações do perfil lipídico sob os diferentes regimes terapêuticos.

\section{MÉTODOS}

\section{Casuística}

Foram selecionados pacientes de ambos os sexos ( $n=20)$ com doença arterial coronária comprovada angiograficamente, em condição clínica estável ${ }^{14}$, sob uso de estatinas há pelo menos três meses, com níveis de LDL-colesterol $<100 \mathrm{mg} / \mathrm{dl}$, e sem previsão de procedimentos de revascularização percutânea ou cirúrgica. Foram excluídos os pacientes com alterações da função hepática (AST $\geq 2,5$ vezes o limite superior da normalidade), renal (creatinina $\geq 2 \mathrm{mg} / \mathrm{dl}$ ), aqueles com comorbidades não controladas, como diabetes melito [hemoglobina glicada $(\mathrm{HbA} 1 \mathrm{c})>7 \%$ ], hipotireoidismo $(\mathrm{TSH}>8 \mu \mathrm{U} / \mathrm{ml}$ ), obesidade (índice de massa corporal $>35 \mathrm{~kg} / \mathrm{m}^{2}$ ) ou com níveis de triglicérides $\geq 400 \mathrm{mg} / \mathrm{dl}$. Foram também excluídos pacientes com dislipidemias genéticas, insuficiência cardíaca classe funcional III/IV da New York Heart Association (NYHA) ${ }^{15}$, os portadores do vírus da imunodeficiência humana, os intolerantes aos fármacos do estudo, e aqueles em uso de inibidores de bomba de prótons.

O protocolo do estudo foi aprovado pelo Comitê de Ética em Pesquisa da Universidade Federal de São Paulo e os pacientes foram incluídos após obtenção do termo de consentimento livre e esclarecido.

O desenho do estudo é apresentado nas Figuras 1 e 2. Inicialmente os pacientes foram selecionados para uma visita médica, em que foram obtidos dados clínicos e colhidas amostras de sangue para exames bioquímicos. Após 7 dias (D) foram avaliados os critérios de inclusão/exclusão, e os pacientes elegíveis tiveram a estatina em uso suspensa por uma semana, sendo agendada a visita 1 (V1) com o paciente internado por 24 horas (Figuras 1 e 2). O período proposto de suspensão do hipolipemiante, superior a cinco meias- 


\section{Desenho do estudo: seleção e estratégias}

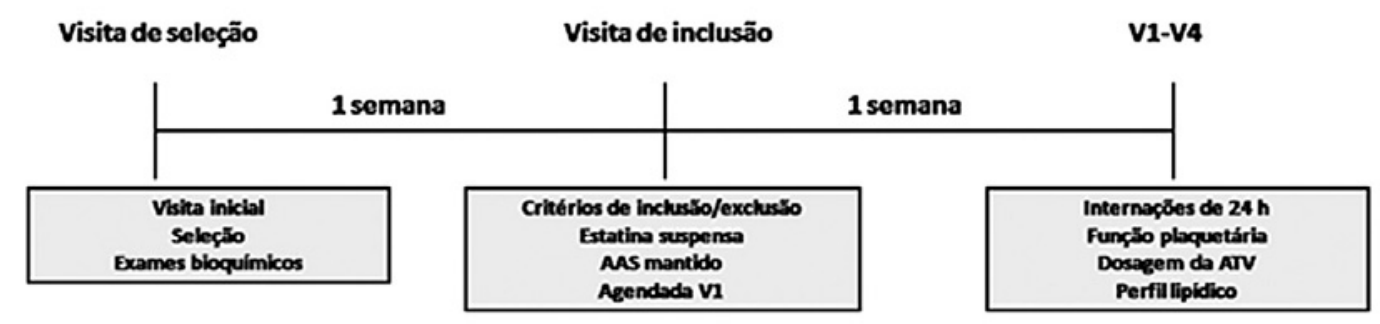

Figura 1 - Desenho do estudo. Período de seleção e estratégias iniciais do estudo. Na visita de seleção os pacientes foram submetidos a avaliação clínica, coleta de exames laboratoriais e mantiveram o uso de ácido acetilsalicílico e estatina em uso prévio. Uma semana após, os critérios de inclusão/exclusão foram confirmados e o paciente foi orientado a manter o ácido acetilsalicílico e suspender a estatina, sendo agendadas as internações ( $\mathrm{V} 1$ a $\mathrm{V} 4)$. AAS = ácido acetilsalicílico; $\mathrm{ATV}=$ atorvastatina; $\mathrm{V}=$ visita.

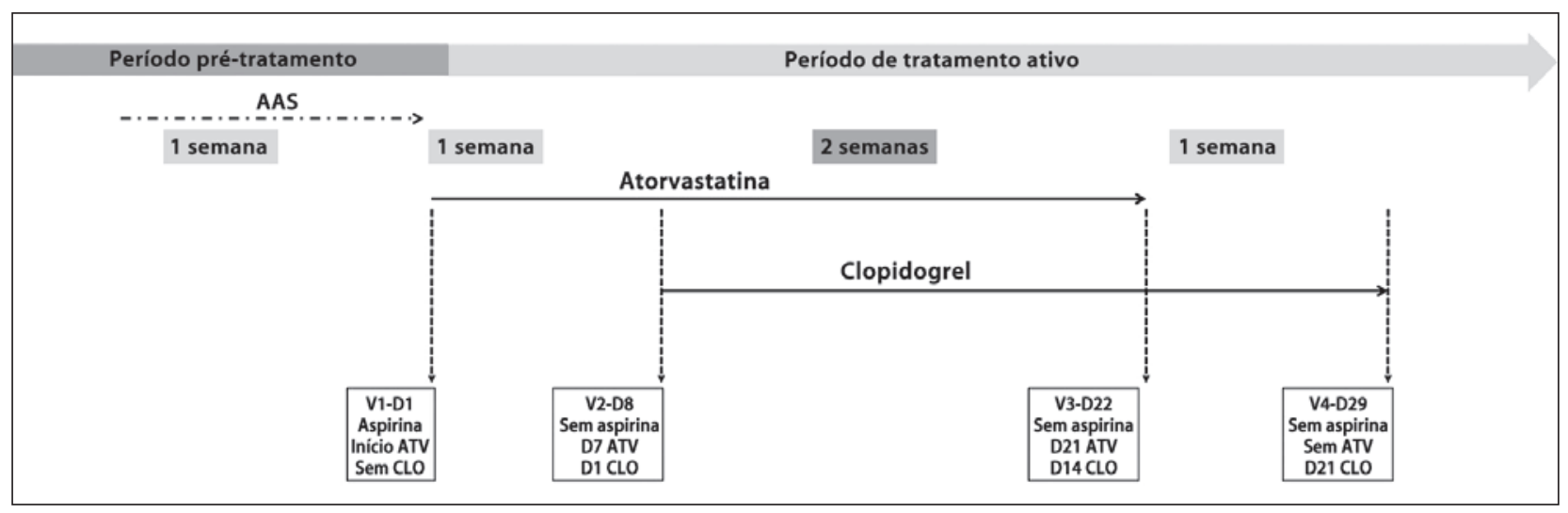

Figura 2 - Desenho do estudo. Período de tratamento ativo, em que os pacientes foram submetidos a quatro períodos de internação por 24 horas (V1 a V4) para receber tratamentos com atorvastatina $80 \mathrm{mg}$ e/ou clopidogrel $75 \mathrm{mg}$. Em todas as visitas foram analisados o perfil lipídico, a função plaquetária e os níveis plasmáticos em 24 horas da atorvastatina. Em V1, os pacientes iniciaram atorvastatina 80 mg e foram orientados a suspender o ácido acetilsalicílico; em V2, foi acrescido clopidogrel 75 mg à atorvastatina 80 mg; em V3, foi estudado o efeito pleno de ambos os fármacos, sendo suspensa a atorvastatina no D23; e em V4, foi analisado o efeito isolado do clopidogrel. AAS = ácido acetilsalicílico; ATV = atorvastatina; $C L O=$ clopidogrel; $D=$ dia; $V=$ visita.

vidas dos fármacos mais potentes, evita mudança no perfil lipídico, e consequente ativação plaquetária, o que seria indesejado em pacientes em prevenção secundária.

A Figura 2 demonstra os quatro períodos de tratamento ativo do estudo. Em todas as visitas (V1 a V4) os pacientes foram internados por um período de 24 horas, receberam as medicações do estudo, além da terapia concomitante usual, e realizaram todos os procedimentos, de acordo com o protocolo.

Em V1 os pacientes tiveram o ácido acetilsalicílico suspenso e iniciaram atorvastatina $80 \mathrm{mg}$ (D1), que foi mantida até V3 (D22). Em V2 (D8), uma semana após $\mathrm{V} 1$, além da atorvastatina foi introduzido clopidogrel $75 \mathrm{mg}$, mantido até o término do estudo (V4, D29). V3 (D22) ocorreu duas semanas após V2 (D8); e em V4 (D29) os pacientes faziam uso isolado do clopidogrel (Figura 2). Em todas as visitas os medicamentos foram fornecidos aos pacientes.
Nessas visitas foram obtidas amostras de sangue para estudo dos níveis séricos da atorvastatina e dos efeitos na adesão/agregação plaquetária sob terapia com atorvastatina/clopidogrel isolada ou em associação (Figura 2).

Todos os pacientes receberam os medicamentos do estudo no mesmo horário, pela manhã, em jejum, após a coleta de sangue basal. Nos dias de estudos farmacocinéticos, a medicação foi administrada na unidade de internação. Houve contagem dos comprimidos retornados a cada visita.

\section{Exames laboratoriais}

Esses exames foram realizados no laboratório da Associação Fundo de Incentivo à Psicobiologia (AFIP) da Universidade Federal de São Paulo (Unifesp). O colesterol total foi analisado por método enzimático fotométrico; o colesterol de lipoproteína de alta densi- 
Pinheiro LFM, et al. Efeitos da Terapia Combinada Atorvastatina e Clopidogrel na Biodisponibilidade da Estatina e na Função Plaquetária em Pacientes com Doença Coronária Estável. Rev Bras Cardiol Invasiva. 2010;18(2):185-92.

dade (HDL-colesterol), por método homogêneo; e os triglicérides, por método enzimático colorimétrico. O LDL-colesterol foi estimado pela equação de Friedewald. ${ }^{16}$ Todas as análises foram automatizadas (ADVIA 1650 ou 2400 Chemistry System, Siemens, Estados Unidos).

A HbA1c foi dosada por cromatografia líquida de alta resolução (HPLC) e as apolipoproteínas (Apo) A1 e B100, por nefelometria (Beckmann Array360, Alemanha).

\section{Estudos farmacocinéticos}

Os pacientes, após jejum de 12 horas, fizeram as coletas nos horários basal e depois de 0,5, 1, 1,5, 2, $2,5,3,4,6,8,10,12,16$ e 24 horas da administração das doses dos fármacos do estudo.

O nível plasmático da atorvastatina foi determinado por cromatografia líquida de alta performance (HPLC, Agilent, Alemanha) e espectrometria de massa (API4000, Sciex/Applied Biosystems, Canadá) $)^{3,4}$, utilizando-se os padrões de atorvastatina (Sigma-Aldrich, Estados Unidos), de forma a se obter a curva de concentração do fármaco, cálculo das áreas sob as curvas (AUC) de Cmax e TMax. As concentrações da atorvastatina foram analisadas sem (V1) ou na presença de clopidogrel (V2 e V3) (Figura 2). As determinações farmacocinéticas foram realizadas no Instituto Galeno, em Campinas (SP).

\section{Adesão e agregação plaquetárias}

Foram realizadas com os pacientes em jejum, pela manhã, em sangue total, utilizando o aparelho Impact R (DiaMed, Suíça), que testa a função plaquetária (adesão e agregação) em condições próximas à fisiológica, utilizando a técnica do cone e placa. ${ }^{17,18}$ Foram utilizados testes para o ácido araquidônico, para testar o ácido acetilsalicílico, e adenosina difosfato para testar o clopidogrel. As plaquetas aderidas e o tamanho dos agregados foram quantificados por um analisador de imagens e seus resultados foram expressos como \% (adesão) da superfície coberta pelos agregados, e pela medida da área em $\mu \mathrm{m}^{2}$ (agregação). ${ }^{17}$

\section{Análise estatística}

Variáveis categóricas são apresentadas como n (\%), as numéricas como médias e erros padrão das médias (EPM). Os dados entre as visitas (lípides, concentração de atorvastatina e função plaquetária) foram comparados por análise de variância (ANOVA) com medidas repetidas, e pós-teste de Tukey. Foi usado o teste $t$ de Student pareado para comparar os valores lipídicos no período basal com V1. O nível de significância foi estabelecido para um risco alfa $<5 \%$.

\section{RESULTADOS}

\section{Características da população estudada}

A Tabela 1 mostra as principais características da amostra. No total, foram avaliados 20 pacientes de
TABELA 1

Características clínicas e laboratoriais basais dos pacientes do estudo

\begin{tabular}{lc}
\hline Parâmetro & Valores \\
\hline Idade, anos & $62(2)$ \\
Sexo masculino, n (\%) & $14(70)$ \\
Hipertensos, n (\%) & $20(100)$ \\
Diabéticos, n (\%) & $7(35)$ \\
Colesterol total, mg/dl & $145(6)$ \\
LDL-colesterol, mg/dl & $76(5)$ \\
HDL-colesterol, mg/dl & $44(2)$ \\
Triglicérides, mg/dl & $122(15)$ \\
Peso, kg & $73,4(2,7)$ \\
Índice de massa corporal, kg/m² & $25,5(1,1)$ \\
Circunferência abdominal, cm & $91,6(2)$ \\
Glicose, mg/dl & $110(3,8)$ \\
Creatinina, mg/dl & $1,08(0,08)$ \\
Alanina aminotransferase, U/l & $26(3)$ \\
Creatinofosfoquinase, U/l & $143(31)$ \\
Hemoglobina, g/dl & $14,3(0,3)$ \\
Hematócrito, \% & $42,2(0,9)$ \\
Glóbulos brancos, n/mm ${ }^{3}$ & $7.296(652)$ \\
Plaquetas, n/mm ${ }^{3}$ & $27.8416(12.940)$ \\
&
\end{tabular}

Os valores apresentados correspondem a médias (EPM) ou dados em porcentagem para variáveis categóricas.

ambos os sexos (70\% homens), com média de idade (EPM) de 62 (2) anos, os quais participaram das quatro etapas do protocolo. Apresentavam valor médio (EPM) de LDL-colesterol de 76 (5) $\mathrm{mg} / \mathrm{dl}$, índice de massa corpórea de $25,5(1,1) \mathrm{kg} / \mathrm{m}^{2}$, sendo a totalidade de hipertensos e 35\% de diabéticos.

\section{Perfil lipídico ao longo do estudo}

A Tabela 2 mostra a evolução do perfil lipídico de acordo com os tratamentos. O colesterol total e o LDLcolesterol após uma semana de suspensão da estatina (V1, D1) foram maiores quando comparados aos demais períodos de tratamento $(P<0,0001$ vs. V2, V3 e $\mathrm{V} 4)$. Houve aumento do HDL-colesterol em V2, V3 e V4, comparativamente a V1 $(P<0,0001)$, enquanto os níveis de triglicérides em $\mathrm{V} 3$ foram menores que em V1 e V4 ( $P=0,003)$. Além disso, o perfil lipídico foi significativa e globalmente modificado apenas sete dias após a suspensão da estatina usada previamente (V1), comparativamente à dosagem basal. O aumento dos níveis de colesterol total com a suspensão da estatina foi de 63 (8) mg/dl $(P<0,0001$ vs. basal), o LDL-colesterol se elevou em 70 (8) mg/dl $(P<0,0001$ vs. basal), o HDL-colesterol diminuiu em 16 (2) mg/dl ( $P<0,0001$ vs. basal), e os triglicérides se elevaram em 63 (21) mg/dl ( $P=0,009$ vs. basal). 
Pinheiro LFM, et al. Efeitos da Terapia Combinada Atorvastatina e Clopidogrel na Biodisponibilidade da Estatina e na Função Plaquetária em Pacientes com Doença Coronária Estável. Rev Bras Cardiol Invasiva. 2010;18(2):185-92.

\section{Resultados dos estudos de adesão/agregação plaquetárias}

A Tabela 3 mostra os resultados obtidos para os testes de adesão e agregação plaquetárias nas quatro etapas do estudo. Para a adesão plaquetária, menores valores foram encontrados em V4 (clopidogrel isolado) comparado a $\vee 1$, somente com ácido acetilsalicílico $(P=0,003)$. Para a agregação plaquetária, observouse que em V1 e após sete dias de tratamento com $80 \mathrm{mg}$ de atorvastatina (V2) os valores de agregação plaquetária foram maiores que com a terapia combinada ou com o uso isolado de clopidogrel $(P<0,0001)$.

\section{Estudos farmacocinéticos}

A Tabela 4 mostra os resultados da análise das AUC de Cmax e Tmax obtidas para a atorvastatina após a dose de $80 \mathrm{mg}$, sem uso concomitante de clopidogrel (V1), após a primeira semana de terapia com $80 \mathrm{mg}$ de atorvastatina, com a introdução do clopidogrel (V2), e em V3, correspondendo ao uso de duas semanas de clopidogrel 75 mg e três semanas de atorvastatina $80 \mathrm{mg}$. Os valores encontrados para as AUC de Cmax não diferiram entre as visitas $(P=0,438 ; V 1=V 2=V 3)$. A atorvastatina não foi detectada na $\mathrm{V} 4$ em nenhum paciente analisado (os pacientes nessa visita estavam em uso de clopidogrel e com atorvastatina suspensa há uma semana), pois após um período equivalente a cinco meias-vidas da estatina não se encontram níveis plasmáticos detectáveis do fármaco.
As AUC do TMax da atorvastatina também não diferiram entre as três visitas $(P=0,156 ; \mathrm{V} 1=\mathrm{V} 2=\mathrm{V} 3)$, como mostrado na Tabela 4.

\section{Visita e exames laboratoriais finais (V4)}

Os pacientes toleraram a medicação instituída (atorvastatina $80 \mathrm{mg}$ e clopidogrel $75 \mathrm{mg}$ ) e foi observada aderência de $100 \%$ à medicação do estudo. Não foram registrados eventos adversos durante o curto período de tratamento do estudo e os pacientes não fizeram uso de medicamentos protetores gástricos que pudessem interferir na biodisponibilidade do clopidogrel. Os exames laboratoriais mostrados na Tabela 5 correspondem à utilização isolada do clopidogrel (visita final - V4), após suspensão da atorvastatina por uma semana.

\section{DISCUSSÃO}

Os achados deste estudo sugerem alguns aspectos interessantes e novos. Observamos que, após uma semana de suspensão de estatinas, os valores obtidos para o colesterol total e o LDL-colesterol foram significantemente maiores que os obtidos antes da suspensão da estatina prévia e em todos os tempos após a introdução da atorvastatina $80 \mathrm{mg}$. Houve alteração no perfil lipídico após curto período de suspensão da estatina usada previamente, em pacientes que a usavam de maneira crônica e que apresentavam LDLcolesterol < $100 \mathrm{mg} / \mathrm{dl}$, sugerindo que mecanismos supressores da síntese de colesterol sejam rapidamen-

TABELA 2

Evolução do perfil lipídico dos pacientes ao longo do estudo

\begin{tabular}{lccccc}
\hline Variável & V1 & V2 & V3 & V4 & Valor de P \\
\hline Colesterol total (mg/dl) & $208(11)$ & $141(5)$ & $161(7)$ & $145(6)$ & $<0,0001^{*}$ \\
HDL-colesterol (mg/dl) & $30(1)$ & $37(2)$ & $41(3)$ & $44(2)$ & $<0,0001+$ \\
LDL-colesterol (mg/dl) & $144(10)$ & $80(5)$ & $101(7)$ & $76(5)$ & $<0,0001^{*}$ \\
Triglicérides (mg/dl) & $184(28)$ & $117(13)$ & $93(12)$ & $180(20)$ & $0,003 \neq$
\end{tabular}

$\mathrm{P}<0,05, \mathrm{ANOVA}$ - medidas repetidas, com pós-teste de Tukey.

$* \mathrm{~V} 1>\mathrm{V} 2, \mathrm{~V} 3$ e $\mathrm{V} 4 ;+\mathrm{V} 1<\mathrm{V} 2, \mathrm{~V} 3$ e $\mathrm{V} 4$; $\neq \mathrm{V} 3<\mathrm{V} 1$ e V4.

HDL-colesterol = colesterol de lipoproteína de alta densidade; LDL-colesterol = colesterol de lipoproteína de baixa densidade; $V=$ visita

TABELA 3

Adesão e agregação plaquetárias de acordo com a terapia instituída

\begin{tabular}{lccccc}
\hline Parâmetro & V1 & V2 & V3 & V4 & Valor de P \\
\hline Adesão, \% & $5,25(0,64)$ & $3,14(1,00)$ & $2,72(0,64)$ & $1,30(0,34)$ & $0,003^{*}$ \\
Agregação, Hm $^{2}$ & $61,23(4,01)$ & $57,37(4,91)$ & $30,10(2,96)$ & $29,54(1,60)$ & $<0,0001+$ \\
\hline
\end{tabular}

Valores expressos como médias (EPM). P < 0,05 (ANOVA - medidas repetidas, com pós-teste de Tukey).

$* \mathrm{~V} 4<\mathrm{V} 1 ;+\mathrm{V} 1$ e $\mathrm{V} 2>\mathrm{V} 3$ e $\mathrm{V} 4$.

$\mathrm{V}=$ visita. 
TABELA 4

Valores obtidos para as AUC de CMax $(\mu \mathrm{M} / \mathrm{l})$

e TMax (hora) da atorvastatina de acordo com as visitas

\begin{tabular}{lcc}
\hline Visita & Cmax & Tmax \\
\hline V1 & $82,60(23,96)$ & $2,28(0,58)$ \\
V2 & $164,22(59,64)$ & $1,50(0,19)$ \\
V3 & $145,81(47,99)$ & $1,28(0,19)$ \\
V4 & Indetectável & Indetectável
\end{tabular}

Valores expressos como médias (EPM), para atorvastatina na dose diária de $80 \mathrm{mg}$ isolada ou associada a clopidogrel $75 \mathrm{mg} /$ dia. Não foram encontradas diferenças entre CMax e TMax entre as visitas $(\mathrm{V} 1=\mathrm{V} 2=\mathrm{V} 3)(\mathrm{ANOVA}-$ medidas repetidas).

CMax = concentração máxima; TMax = tempo para alcançar CMax; $V$ = visita.

TABELA 5 Perfil laboratorial na visita final (V4)

\begin{tabular}{lc}
\hline Parâmetro & Valores \\
\hline Colesterol total, mg/dl & $145(6)$ \\
LDL-colesterol, mg/dl & $76(5)$ \\
HDL-colesterol, mg/dl & $44(2)$ \\
Triglicérides, mg/dl & $180(20)$ \\
Glicose, mg/dl & $121(15)$ \\
Creatinina, mg/dl & $1,02(0,07)$ \\
Alanino aminotransferase, U/l & $27(3)$ \\
Creatinofosfoquinase, U/l & $81(14)$ \\
Hemoglobina, g/l & $14(0,5)$ \\
Hematócrito, \% & $41,2(1,3)$ \\
Glóbulos brancos, $\mathrm{n} / \mathrm{mm}^{3}$ & $6.679(594)$ \\
Plaquetas, $\mathrm{n} / \mathrm{mm}^{3}$ & $275.444(10.577)$ \\
\end{tabular}

Valores expressos como médias (EPM).

HDL-colesterol $=$ colesterol de lipoproteína de alta densidade; $L D L$-colesterol $=$ colesterol de lipoproteína de baixa densidade; $\mathrm{V}=$ visita.

te modificados, e que possa haver rápido aumento da atividade da HMG-CoA redutase, capaz de explicar as alterações precoces observadas. Nesse curto período, não foram observadas alterações hepáticas ou musculares, demonstrando boa tolerabilidade para os fármacos.

Os estudos de adesão e agregação plaquetárias sugeriram que o clopidogrel foi eficiente como antiplaquetário, em uso concomitante com a atorvastatina em dose alta, e aparentemente não houve interação desfavorável entre esses fármacos, dado que não tinha sido verificado na literatura, a não ser com doses mais baixas de estatinas. ${ }^{19,20}$ Avaliamos pacientes com doença arterial coronária estável, dos quais 52\% foram sub- metidos a implante de stents há mais de 180 dias, em uso de ácido acetilsalicílico isolado, para avaliar, em uma condição que não oferecesse risco, as modificações laboratoriais decorrentes da administração isolada ou em combinação desses fármacos. O ácido acetilsalicílico foi suspenso para que pudéssemos analisar os efeitos isolados do tienopiridínico na função plaquetária.

O estudo farmacocinético mostrou que os níveis de atorvastatina não se alteraram de forma significativa após a adição de clopidogrel. Como o uso desses fármacos tem sido preconizado de forma cada vez mais frequente em portadores de doença coronária, sobretudo nas síndromes coronárias agudas ou após intervenções percutâneas, sua utilização nos pareceu segura, com base nos resultados até aqui obtidos.

A dupla terapia antiplaquetária com ácido acetilsalicílico e clopidogrel reduz complicações aterotrombóticas em pacientes que são submetidos a intervenção coronária percutânea com implante de stent, porém existe grande variabilidade individual de respostas, permitindo a ocorrência de eventos aterotrombóticos mesmo entre indivíduos tratados. ${ }^{18,21-25}$ A maior parte dos estudos que avaliou a função plaquetária o fez com casuística pequena e utilizando um único teste. Apesar de não haver consenso sobre o melhor teste, o estudo POPULAR ${ }^{23}$ demonstrou que nem todos são bons preditores de eventos aterotrombóticos, incluindo trombose dos stents, em pacientes tratados com clopidogrel e submetidos a intervenção coronária percutânea. A alta reatividade plaquetária avaliada pelos métodos de agregometria, VerifyNow e Plateletworks, foi associada com eventos aterotrombóticos, o que não foi verificado com os testes baseados na força de cisalhamento (IMPACT-R e PFA). Porém o diabetes melito e a disfunção ventricular esquerda, também preditores de eventos aterotrombóticos após implante de stent, podem ser confundidores. ${ }^{19,20}$ Até a obtenção de evidências mais robustas, nossa prática clínica não deve ser guiada por esses métodos. ${ }^{19,20}$ É senso comum a prescrição simultânea de clopidogrel e atorvastatina após evento coronário ou implante de stents. O clopidogrel induz a alteracões irreversíveis na ligação da adenosina difosfato plaquetária com o receptor P2Y12, com consequente inibição da adesão e agregação plaquetárias. ${ }^{3}$ Essa modificação do receptor adenosina difosfato plaquetário é tempo- e dose-dependente, provoca inibição cumulativa da agregação e lenta recuperação da função plaquetária após a retirada do fármaco. ${ }^{19,22-25}$

O clopidogrel, por ser pródroga, requer metabolismo hepático pelo citocromo P450 (isoenzima 3A4) para gerar seu metabólito ativo ${ }^{4}$, e a administração simultânea de atorvastatina em concentrações equimolares in vitro inibe o metabolismo do clopidogrel em mais de $90 \%{ }^{19}$, como este também pode ser inibido por antifúngicos, imunossupressores e inibidores de protease (todos substratos da isoenzima 3A4). ${ }^{20,26}$ 
Pinheiro LFM, et al. Efeitos da Terapia Combinada Atorvastatina e Clopidogrel na Biodisponibilidade da Estatina e na Função Plaquetária em Pacientes com Doença Coronária Estável. Rev Bras Cardiol Invasiva. 2010;18(2):185-92.

Ainda é controversa a hipótese de interação clopidogrel-estatina ${ }^{22-25,27,28}$ na função plaquetária. A existência de vários métodos para avaliação dos efeitos antiplaquetários induzidos pelo clopidogrel e o fato de os efeitos do clopidogrel serem tempo- e dose-dependentes e de a maioria dos estudos utilizar doses baixas de estatinas ou doses de manutenção do clopidogrel são limitações. ${ }^{4,21}$ É possível que os efeitos inibitórios discretos das estatinas tenham sido superestimados pelos desenhos dos estudos.

\section{Limitações do estudo}

Nosso estudo tem limitações, como casuística pequena e avaliação de curto prazo e com dose de manutenção do clopidogrel. Doses mais elevadas de clopidogrel podem ter impacto maior na biodisponibilidade da estatina.

\section{CONCLUSÃO}

O estudo examinou as interações farmacocinéticas entre o antiplaquetário tienopiridínico mais utilizado e uma das estratégias hoje disponível para se atingir metas adequadas de LDL-colesterol. Os resultados obtidos sugerem que doses plenas da atorvastatina possam ser utilizadas em associação ao clopidogrel, propiciando efetividade nos controles hipolipemiante e antiplaquetário em populações de alto risco.

\section{SUPORTE FINANCEIRO}

O estudo recebeu suporte financeiro da Fundação de Amparo à Pesquisa do Estado de São Paulo (FAPESP2008/55501-6), Luiz Fernando Muniz Pinheiro recebeu bolsa de pós-graduação do Conselho Nacional de Ciência e Tecnologia (CNPq), e Soraia Kani Kasmas e Carolina Nunes França receberam bolsas da FAPESP.

\section{CONFLITO DE INTERESSES}

Os autores declararam inexistência de conflito de interesses relacionado a este manuscrito.

\section{REFERÊNCIAS}

1. Gurbel PA, Lau WC, Bliden KP, Tantry US. Clopidogrel resistance: implications for coronary stenting. Curr Pharm Des. 2006;12(10):1261-9.

2. Neubauer $\mathrm{H}$, Mugge A. Thienopyridines and statins: assessing a potential drug-drug interaction. Curr Pharm Des. 2006;12(10):1271-80.

3. Mach F, Senouf D, Fontana P, Boehlen F, Reber G, Daali $Y$, et al. Not all statins interfere with clopidogrel during antiplatelet therapy. Eur J Clin Invest. 2005;35(8):476-81.

4. Lau WC, Waskell LA, Watkins PB, Neer CJ, Horowitz K, Hoop AS, et al. Atorvastatin reduces the ability of clopidogrel to inhibit platelet aggregation: a new drug-drug interaction. Circulation. 2003;107(1):32-7.

5. Savi P, Pereillo JM, Uzaiaga MF, Combalbert J, Picard C, Malfrand JP, et al. Identification and biological activity of the active metabolite of clopidogrel. Thromb Haemost. 2000;84(5):891-6.
6. Ayalasomayajula SP, Vaidyanathan S, Kemp C, Prasad P, Balch A, Dole WP. Effect of clopidogrel on the steady-state pharmacokinetics of fluvastatin. J Clin Pharmacol. 2007; 47(5):613-9.

7. Eikelboom JW, Weitz JI, Budaj A, Zhao F, Copland I, Maciejewski $\mathrm{P}$, et al. Clopidogrel does not suppress blood markers of coagulation activation in aspirin-treated patients with non-ST-elevation acute coronary syndromes. Eur Heart J. 2002;23(22):1771-9.

8. Cassar K, Bachoo P, Ford I, Greaves M, Brittenden J. Clopidogrel has no effect on D-dimer and thrombin-antithrombin III levels in patients with peripheral arterial disease undergoing peripheral percutaneous transluminal angioplasty. J Vasc Surg. 2005;42(2):252-8.

9. Lim MJ, Spencer FA, Gore JM, Dabbous OH, Agnelli G, Klive-Rogers EM, et al. Impact of combined pharmacologic treatment with clopidogrel and a statin on outcomes of patients with non-ST-segment elevation acute coronary syndromes: perspectives from a large multinational registry. Eur Heart J. 2005;26(11):1063-9.

10. Heestermans AA, Van Werkum JW, Schomig E, ten Berg JM, Taubert D. Clopidogrel resistance caused by a failure to metabolize clopidogrel into its metabolites. J Thromb Haemost. 2006;4(5):1143-5.

11. Kajinami K, Brousseau ME, Ordovas JM, Schaefer EJ. CYP3A4 genotypes and plasma lipoprotein levels before and after treatment with atorvastatin in primary hypercholesterolemia. Am J Cardiol. 2004;93(1):104-7.

12. Farid NA, Payne CD, Small DS, Winters KJ, Ernest CS 2nd, Brandt JT, et al. Cytochrome P450 3A inhibition by ketoconazole affects prasugrel and clopidogrel pharmacokinetics and pharmacodynamics differently. Clin Pharmacol Ther. 2007;81(5):735-41.

13. Jilma-Stohlawetz $P$, Horvath $M$, Eichelberger B, Koren D, Jilma B, Panzer S. Platelet function under high-shear conditions from platelet concentrates. Transfusion. 2008;48(1):129-35.

14. Gibbons RJ, Abrams J, Chatterjee K, Daley J, Deedwania PC, Douglas JS, et al. ACC/AHA 2002 guideline update for the management of patients with chronic stable angina summary article: a report of the American College of Cardiology/ American Heart Association Task Force on Practice Guidelines (Committee on the Management of Patients With Chronic Stable Angina). Circulation. 2003;107(1):149-58.

15. Hunt SA. ACC/AHA 2005 guideline update for the diagnosis and management of chronic heart failure in the adult: a report of the American College of Cardiology/American Heart Association Task Force on Practice Guidelines (Writing Committee to Update the 2001. Guidelines for the Evaluation and Management of Heart Failure). J Am Coll Cardiol. 2005; 46(6): 1 1-82.

16. Friedewald WT, Levy RI, Fredrickson DS. Estimation of the concentration of low-density lipoprotein cholesterol in plasma, without use of the preparative ultracentrifuge. Clin Chem. 1972;18(6):499-502.

17. Morrison A, Hornsey VS, Prowse CV, Macgregor IR. Use of the DiaMed Impact $\mathrm{R}$ to test platelet function in stored platelet concentrates. Vox Sang. 2007;93(2):166-72.

18. Sibbing D, Braun S, Morath T, Mehilli J, Vogt W, Schomig A, et al. Platelet reactivity after clopidogrel treatment assessed with point-of-care analysis and early drug-eluting stent thrombosis. J Am Coll Cardiol. 2009;53(10):849-56.

19. Clarke TA, Waskell LA. The metabolism of clopidogrel is catalysed by human cytocrome P450 $3 \mathrm{~A}$ and is inhibited by atorvastatin. Drug Metab Dispos. 2003;31(1):53-9.

20. Angiolillo DJ, Alfonso F. Clopidogrel-statin interaction. Myth or reality? J Am Coll Cardiol. 2007;50(4):296-8. 
Pinheiro LFM, et al. Efeitos da Terapia Combinada Atorvastatina e Clopidogrel na Biodisponibilidade da Estatina e na Função Plaquetária em Pacientes com Doença Coronária Estável. Rev Bras Cardiol Invasiva. 2010;18(2):185-92.

21. Saw J, Brennan DM, Steinhubl SR, Bhatt DL, Mak KH, Fox $\mathrm{K}$, et al.. Lack of evidence of a clopidogrel-statin interaction in the CHARISMA trial. J Am Coll Cardiol. 2007;50(4):291-5.

22. Marcucci R, Gori AM, Paniccia R, Giusti B, Valente S, Giglioli C, et al. Cardiovascular dead and nonfatal myocardial infarction in acute coronary syndrome patients receiving coronary stenting are predicted by residual platelet reativity to ADP detected by a point-of-care assay. Circulation. 2009; 119(2):237-42.

23. Breet NJ, van Werkum JW, Bouman HJ, Kelder JC, Ruven $\mathrm{HS}$, Bal ET, et al. Comparison of platelet function tests in predicting clinical outcome in patients undergoing coronary stent implantation. JAMA. 2010;303(8):754-62.

24. Van Werkun JW, Heestermans AA, Zomer AC, Kelder JC, Suttorp MJ, Rensing BJ, et al. Predictors of coronary stent thrombosis. J Am Coll Cardiol. 2009;53(16):1399-409.
25. Bonello L, Camoin-Jau L, Armero S, Com O, Arques S, Burignat-Bonello C, et al. Tailored clopidogrel loading dose according to platelet reactivity monitoring to prevent acute and subacute stent thrombosis. Am J Cardiol. 2009;103(1): 5-10.

26. Busti AJ, Bain AM, Hall RG 2nd, Bedimo RG, Leff RD, Meek $C$, et al. Effects of atazanavir/ritonavir or fosamprenavir/ ritonavir on the pharmacokinetics of rosuvastatin. J Cardiovasc Pharmacol. 2008;51(6):605-10.

27. Jennings LK. Variability in platelet response to the antiplatelet agents aspirin and clopidogrel. Mechanisms, measurement and clinical relevance. Crit Path Cardiol. 2009;8(1):20-8.

28. Schwammenthal Y, Tsabari R, Shenkman B, Schwartz R, Matetzky $S$, Lubetsky $A$, et al. Aspirin responsiveness in acute brain ischaemia: association with stroke severity and clinical outcome. Cerebrovasc Dis. 2008;25(4):355-61. 